\title{
Arterial blood pressure of term newborns during the first week of life
}

M.C.V.A. Nascimento ${ }^{1}$ C.C. Xavier ${ }^{2}$ and E.M.A. Goulart²
${ }^{1}$ Departamento de Pediatria, Fundação Educacional Lucas Machado, Faculdade de Ciências M édicas de M inas Gerais, Belo Horizonte, M G, Brasil ${ }^{2}$ Departamento de Pediatria, Faculdade de Medicina, Universidade Federal de Minas Gerais, Belo Horizonte, MG, Brasil

\section{Correspondence}

M.C.V.A. Nascimento

Rua Jorge Marine, 79

30320-550 Belo Horizonte, MG

\section{Brasil}

E-mail: nascimen@globalsite.com.br

Received October 17, 2001

Accepted June 14, 2002

\section{Abstract}

The progressive behavior of the blood pressure of term newborns during the first week of life was assessed by the simultaneous use of oscillometric and Doppler methods. A total of 174 term neonates born at the Municipal Hospital Odilon Behrens in Belo Horizonte, from March 1996 to February 1997, were prospectively assessed. The oscillometric and Doppler ultrasonic methods were simultaneously used for four consecutive recordings obtained at $12 \pm 6,24 \pm 6$ and 72 $\pm 24 \mathrm{~h}$ and on the 7 th \pm 1 day of life. The combined use of the two methods simplified the procedure, with automatic cuff inflation and deflation, and speed was properly controlled with an automatic pressure monitor. The procedure was performed using a Y-connection to the mercury sphygmomanometer, with blood pressure being recorded with an automatic device and systolic blood pressure being measured simultaneously by Doppler ultrasound. The newborns were awake, not crying and in the supine position. A statistically significant increase in systolic and diastolic blood pressure was observed between the first and second, and the third and fourth measurements by Doppler and oscillometric methods. No significant correlation between birth weight, length, ponderal index and blood pressure was observed. The technique used represents a simpler and more accurate procedure for blood pressure measurement.
Key words

- Blood pressure

- Doppler method

- O scillometric method

- Term newborn

\section{Introduction}

As measurement methods improve, more appropriate reference values for blood pressure of newborns are necessary. Normal variations may be difficult to interpret due to different measurement techniques, variation in birth weight and gestational age, rapid change in body size and marked increase in blood pressure in the first hours and days of life (1). The definition of normal blood pres- sure values is complex and, from a clinical point of view, it is difficult to establish a threshold between "normal" and "abnormal". Therefore, measurements made with an adequate methodology reflecting the blood pressure behavior of newborns are necessary.

The objective of the present study was to describe the behavior of blood pressure in term newborns simultaneously measured by oscillometric and Doppler methods during the first week of life. 


\section{Material and Methods}

The study was approved by the Research Ethics Committee, Federal University of Minas Gerais, and by the Research Ethics Committee of the Municipal Hospital Odilon Behrens. Informed consent was obtained from the parents or persons responsible for the newborn. This was a longitudinal and prospective study conducted on 174 term neonates, appropriate for gestational age (2), in good clinical conditions, born at the Municipal Hospital Odilon Behrens, Belo Horizonte, from March 1, 1996 to February 28, 1997. The exclusion criteria were severe renal or cardiac congenital malformations, clinical progression making planned recordings impossible or interfering with the measurements, and newborns whose mothers used drugs known to be hypotensive or hypertensive agents during pregnancy.

Blood pressure of newborns was recorded four times, i.e., at $12 \pm 6,24 \pm 6$ and $72 \pm 24$ $\mathrm{h}$ of life during hospitalization, and on the 7 th \pm 1 day of life at the outpatient clinic.

The oscillometric method using the noninvasive automatic pressure device DX-2710 (Dixtal, Manaus, AM, Brazil) and the Doppler method using a Doppler Vascular DV 20 device (Microem, Ribeirão Preto, SP, Brazil) (Figure 1) were applied simultaneously to measure blood pressure. Meas-

Figure 1. Technique used for the simultaneous determination of blood pressure in newborns by oscillometric and Doppler methods.

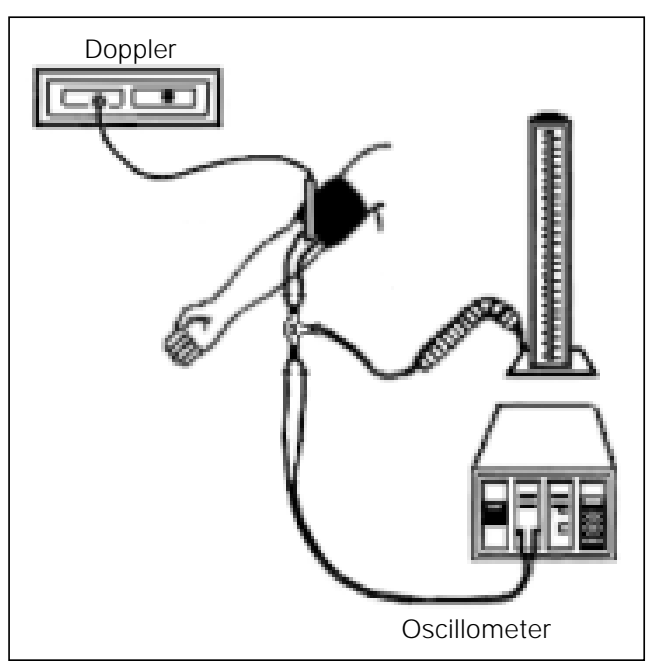

urements were made when the newborns were awake, in the supine position, sucking a pacifier and not crying, not at feeding time, and their axillary temperature was usually between 36.5 and $37.4^{\circ} \mathrm{C}$.

The cuff was wrapped around the right arm and connected to a conjugated system with two Ys and one three-way arm (Figure 1). The cuff tubes were connected to the first $\mathrm{Y}$ on one side, and to the three-way arm on the other. The three-way arm was connected to a wallmounted mercury sphygmomanometer, which was calibrated frequently. The oscillometer tubes were connected to the other Y.

The Doppler probe was placed on the cubital fossa of the arm over the brachial artery, with conducting gel. The arm was extended and immobilized by the examiner's hand. Next, the Doppler equipment was switched on and the brachial artery flow was auscultated. The oscillometer was turned on with the help of the assistant, and the cuff was automatically inflated and deflated. During inflation of the cuff, the brachial artery flow sound was not heard when the artery was completely occluded, and was heard only during deflation. This moment, when the sound is heard, corresponds to the systolic pressure determined by the Doppler method, recorded by the mercury sphygmomanometer connected to the $\mathrm{Y}$ and visualized by the examiner, whose gaze is directed at the top of the mercury column (3-7).

Next, systolic, mean arterial and diastolic blood pressures and heart rate were automatically recorded by the oscillometric method on the digital display of the pressure monitor (8-11).

The cuffs were chosen according to brachial length and brachial circumference, considering a cuff width/brachial circumference ratio of $0.4-0.5$, and completely wrapped around the arm (11-13).

The simultaneous use of oscillometric and Doppler methods was appropriate since each measuring technique made the other easier. Moreover, the two methods are com- 
parable, presenting no great discrepancies and providing accurate measurements.

All blood pressure measurements were made by the senior author, who was helped by an assistant only to switch on the oscillometric device and keep the child well positioned.

Anthropometric indicators and birth weight were checked and correlation coefficients between these variables and blood pressure were calculated.

The sample size was calculated using the True Epistat software (14). In the population mean estimate, a $95 \%$ confidence interval (CI) of 1- $\alpha$ and a maximum difference between the true population mean and the sample mean of $2 \mathrm{mmHg}$ were taken into account. Based on the study by De Swiet et al. (15), which assessed 42 newborns, the systolic blood pressure variance of 100 , by the Doppler method, was considered (standard deviation of $10.0 \mathrm{mmHg}$ ). The sample size calculated by the software resulted in 146 infants, and $30 \%$ of this value was added as a safety margin.

Data were stored and analyzed using the Epi-Info version 6.02 software (16). Analysis of variance was used to compare means. When means were not comparable to heterogeneous variances, median comparison was applied. In order to study the correlation between two continuous variables, the correlation coefficient ( $\mathrm{r}$ ) and its $95 \% \mathrm{CI}$ were calculated. In the blood pressure study, the statistical technique of longitudinal data anal- ysis (17) was used in the four records since measurements were made on the same neonate. A 95\% CI was used. The statistical significance limit considered was $5 \%(\mathrm{P}<0.05)$.

\section{Results}

Of 174 term newborns studied, 78 (44.8\%) were males. Birth weight ranged from 2,280 to $3,880 \mathrm{~g}$ (mean $=3,095 \pm 313 \mathrm{~g}$ and median $=$ $3,085 \mathrm{~g}), 3.4 \%$ were low-birth weight newborns, and $12.6 \%$ weighed $\geq 3,500 \mathrm{~g}$. The 5-min Apgar score was $\geq 7$ in $100 \%$ of them.

As to mode of delivery, 104 babies were born by vaginal delivery (59.8\%), 64 (36.8\%) by cesarean section and $6(3.4 \%)$ by forceps. A total of 104 (59.8\%) mothers were submitted to local anesthesia, 61 (35.1\%) to spinal anesthesia, $5(2.9 \%)$ to epidural anesthesia, and $3(1.7 \%)$ did not receive any type of anesthesia. Thirty-one mothers (17.8\%) were diagnosed as suffering from maternal hypertensive disease at the time of hospital admission, 4 of them (13\%) with pregnancyinduced hypertension. We considered maternal hypertensive disease to be present when a diagnosis of arterial hypertension was reported in the obstetric case notes and was confirmed by the recording of at least one maternal systolic blood pressure value higher than $140 \mathrm{mmHg}$ and/or a diastolic blood pressure value higher than $90 \mathrm{mmHg}$, with no use of antihypertensive agents.

Table 1 presents the newborn blood pres-

Table 1. Systolic (SBP), mean arterial (MAP) and diastolic (DBP) blood pressure measured by the oscillometric method and SBP by Doppler of term newborns during the first week of life.

\begin{tabular}{|c|c|c|c|c|c|}
\hline \multirow[t]{2}{*}{ Age } & \multirow[t]{2}{*}{$\mathrm{N}$} & \multicolumn{3}{|c|}{ Oscillometric method } & \multirow{2}{*}{$\begin{array}{c}\text { Doppler } \\
\text { SBP }\end{array}$} \\
\hline & & SBP & MAP & DBP & \\
\hline $12 \pm 6 h$ & 174 & $79.7 \pm 12.9(\mathrm{~A})^{*}$ & $56.5 \pm 12.3(\mathrm{~A})$ & $42.5 \pm 10.4(\mathrm{~A})$ & $75.5 \pm 11.6(\mathrm{~A})$ \\
\hline $24 \pm 6 h$ & 174 & $83.2 \pm 12.4(\mathrm{~B})$ & $60.2 \pm 11.3(\mathrm{~B})$ & $46.2 \pm 9.8(\mathrm{~B})$ & $79.8 \pm 12.6(\mathrm{~B})$ \\
\hline 3 days $\pm 6 \mathrm{~h}$ & 174 & $83.5 \pm 13.8(\mathrm{~B})$ & $60.8 \pm 11.8(B)$ & $47.8 \pm 12.9(B)$ & $80.7 \pm 12.6(\mathrm{~B})$ \\
\hline $7 \pm 1$ day & 120 & $91.1 \pm 15.3(\mathrm{C})$ & $66.6 \pm 12.8(\mathrm{C})$ & $51.8 \pm 11.4(\mathrm{C})$ & $88.1 \pm 13.4(\mathrm{C})$ \\
\hline
\end{tabular}

Data are reported as means \pm SD and were compared for each method using analysis of variance and a longitudinal data analysis model. *Statistical difference between means $(A \neq B \neq C)$. 
sures (means $\pm \mathrm{SD}$ ) simultaneously measured on four occasions by oscillometric and Doppler methods. Blood pressure levels increased from the first to the second reading $(\mathrm{P}=0.0002)$ and from the third to the fourth reading $(\mathrm{P}<0.0001)$, but not from the second to the third reading $(P=0.98)$. This result was obtained by sequential data analysis using a mathematical model of longitudinal data analysis (17), since it was possible to make four blood pressure measurements in the same neonate. The same behavior was observed for diastolic, mean and systolic arterial blood pressure measured by Doppler.

In the first three readings there was no loss to follow-up. In the fourth record, the losses $(\mathrm{N}=54)$ were compared to cases in which blood pressure was checked $(\mathrm{N}=$ 120 ) in terms of sex, birth weight and length. Statistical equivalence was found for both groups $(\mathrm{P}>0.05)$ for all variables.

The correlation coefficients $(r)$ calculated for systolic pressure measured by oscillometric and Doppler methods were 0.80, 0.70, 0.78 and 0.85 for the four consecutive records. These results indicate strong correlations, all of them statistically significant, demonstrating equivalence of the two methods.

No significant correlation coefficients

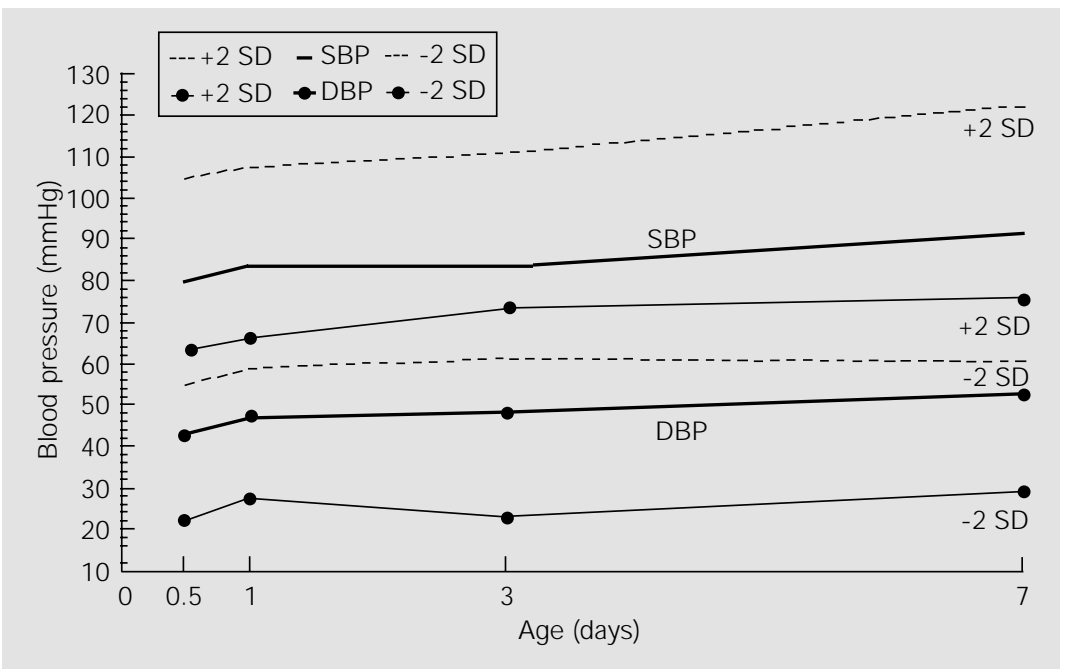

Figure 2. Systolic (SBP) and diastolic (DBP) blood pressure values measured by the oscillometric method (mean $\pm 2 \mathrm{SD}$ ) in term newborns. were obtained between blood pressure and newborn anthropometric data such as weight, length, head circumference and ponderal index (weight/length ${ }^{3}$ ). The correlation coefficients between weight and systolic, mean arterial and diastolic blood pressures measured by the oscillometric method were -0.01 , 0.06 and 0.09 , respectively, and the coefficient between weight and systolic blood pressure measured by Doppler was 0.05 .

No differences in the measurements were observed in the first record when neonates were divided into groups by weight.

No significant correlations were observed between mode of delivery and blood pressure measured by the oscillometric (ANOVA = 0.63, $\mathrm{P}=0.53)$ and Doppler (ANOVA = $0.17, \mathrm{P}=0.84)$ methods or between maternal anesthesia and blood pressure measured by the oscillometric (Kruskal-Wallis $=0.80$, $\mathrm{P}=0.67)$ and Doppler (ANOVA $=0.13$, $\mathrm{P}=0.88$ ) methods.

Also, no significant correlation was observed between neonate sex and blood pressure measured by the oscillometric (ANOVA $=2.99, \mathrm{P}=0.08)$ and Doppler (ANOVA $=$ $0.21, \mathrm{P}=0.65)$ methods.

Figure 2 illustrates the systolic and diastolic blood pressure values measured by the oscillometric method and reported as means $\pm 2 \mathrm{SD}$.

\section{Discussion}

The simultaneous measurement of blood pressure by the Doppler ultrasonic and oscillometric methods allowed us to evaluate blood pressure values in a simpler and more accurate fashion. A recent publication (18) has suggested the use of this methodology in adults, but no study using a similar methodological application to newborns was found in the literature.

The method used to measure blood pressure was simplified by automatic inflation and deflation through a noninvasive pressure monitor at the speed of $2-3 \mathrm{mmHg} / \mathrm{s}$ 
$(18,19)$. This speed is difficult to achieve manually with the conventional mercury sphygmomanometer valves and almost always requires the presence of another examiner. This procedure also permits an adequate immobilization of the arm and positioning of the Doppler probe in the cubital fossa of newborns.

The correlation coefficients between the mean systolic pressure values determined by the oscillometric and Doppler methods over four recordings were $0.80,0.70,0.78$, and 0.85 , respectively, indicating a strong correlation. All data were statistically significant, representing more accurate measurements.

A survey of studies carried out with the Doppler method using at least three readings during the first week of life showed different results. The study by Cona (20) performed on the first, second and third days of life reported higher blood pressure levels than those found in the present investigation. The infants were awake, but the authors did not report if they were calm and not crying. Moreover, no length or brachial circumference measurements were made for a more adequate selection of the cuff to be used. De Swiet et al. (15), taking five readings during the first week of life (third, fourth, fifth, sixth and seventh day of life), and Uhari (21), taking three readings (first, fourth and fifth day of life), found lower blood pressure levels, with means that were significantly different from those obtained here. This fact could be explained by racial differences, since over $90 \%$ of the newborns participating in these two studies were Caucasian. In addition, the measurements were made immediately after the meals, when blood pressure levels are lower (22). Due to the miscegenation of the Brazilian population, we did not classify our population according to race or skin color.

In the present investigation, all blood pressure measurements were made when newborns awoke spontaneously or were nicely awaken by the examiner putting a pacifier in their mouth, or caressing their face. All infants sucked the pacifier and none of them was crying during each recording. Therefore, the mean blood pressure value obtained by the Doppler method on the 7th day was $88.1 \pm 13.4 \mathrm{mmHg}$ in 120 newborns. Comparison with the value obtained in the study by De Swiet et al. (15), carried out on 42 awake newborns $(77.0 \pm 10.0$ $\mathrm{mmHg}$ ), showed a significant difference $(\mathrm{P}<0.0001)$. However, it should be emphasized that in the study by De Swiet et al. (15), $96.5 \%$ of the infants were Caucasian and pressure measurements were made soon after meals. Thus, care should be taken when comparing blood pressure values due to the variety of tools and techniques used.

A recent Brazilian study (23) using an oscillometric technique showed slightly lower levels of systolic, mean and diastolic arterial blood pressure than the present study. No statistically significant difference in two readings that agreed (third and seventh day of life) was observed between the blood pressure values obtained in this and the present study. Nonetheless, for 12-h-old neonates, the mean systolic and diastolic blood pressure values were significantly different $(\mathrm{P}=0.0085)$. The mean systolic blood pressure obtained by Matsuoka et al. (23) for 35 newborns was $73.6 \pm 11.2 \mathrm{mmHg}$ versus $79.7 \pm 12.9 \mathrm{mmHg}$ in the present study.

In a previous investigation (24), assessing blood pressure of 174 newborns with birth weight ranging from 2,280 to $3,880 \mathrm{~g}$, no significant correlation between birth weight and blood pressure was observed. The correlation coefficients (r) between weight and systolic, mean and diastolic arterial blood pressure, measured on average at $12 \pm 6 \mathrm{~h}$ of life by the oscillometric method, were $-0.01,0.06$ and 0.09 , respectively, and the correlation coefficient between weight and systolic blood pressure measured by the Doppler method was 0.05 . However, it should be emphasized that only 6 infants weighing $<2,500 \mathrm{~g}$ and 22 weighing $\geq 3,500 \mathrm{~g}$ were 
evaluated. The results of the present study are similar to those reported by Lieberman (25), Whincup et al. (26) and Taittonen et al. (27).

O'Sullivan et al. (28) evaluated only one blood pressure reading obtained with the oscillometric method on the third or fourth day of life for 248 newborns (mean weight $=$ $3,492.9 \pm 483.8 \mathrm{~g}$; range 2,120 to $4,990 \mathrm{~g}$ ) and reported a positive correlation between systolic blood pressure and birth weight $(\mathrm{r}=$ $0.17, \mathrm{P}=0.006)$.

Uhari (21) reported a statistically significant correlation between systolic blood pressure and birth weight $(\mathrm{r}=0.15, \mathrm{P}<0.05)$, using the same cuff size ( $4 \mathrm{~cm}$ wide x $5 \mathrm{~cm}$ long) in all newborns, but did not describe infant mean weight or weight variability. De Swiet et al. (15) also reported a statistically significant correlation between weight and blood pressure in 71 four-day-old newborns, ranging in weight from 2,800 to $3,800 \mathrm{~g}(\mathrm{r}=$ $0.30, \mathrm{P}<0.0001)$. However, the authors described these coefficients as "low", although statistically significant, and recommended further investigation.

It has been speculated that there might be a correlation between birth weight and subsequent hypertension, and this correlation is stronger in adults than in children and adolescents. Longitudinal data on birth weight and blood pressure during childhood are inconsistent and controversial (26). Determining the validity of the results of crosssectional and longitudinal studies on the relation of birth weight and blood pressure requires well-designed studies, including prospective protocols with comparable techniques used to measure blood pressure, detailed analysis of maternal nutrition and anthropometric data.

The technique used here combining oscillometric and Doppler methods permits simple and accurate blood pressure measurements. The correlation coefficients between blood pressure means measured by the two methods demonstrated a strong correlation.

\section{References}

1. Goble MM (1993). Hypertension in infancy. Pediatric Clinics of North America, 40: 105-122.

2. Lubchenco LO, Hansman C, Dressler M \& Boyd E (1963). Intrauterine growth as estimated from live bom birth-weight data at 24 to 42 weeks of gestation. Pediatrics, 32: 793-800.

3. De Swiet $M$, Fayers $P \&$ Shineboume EA (1976). Blood pressure survey in the population of newborn infants. British Medical J ournal, 2: 9-11.

4. Dweck HS, Reynolds DW \& Cassady G (1974). Indirect blood pressure measurement in newboms. American J oumal of Diseases of Children, 127: 492-494.

5. Elseed AM, Shineboume EA \& J oseph MC (1973). Assessment of techniques for measurement of blood pressure in infants and children. Archives of Disease in Childhood, 48: 932-936.

6. Gordon LS, J ohnson J r PE, Penido J RF, Printup J r C, Dietrick WR \& Bugggs $\mathrm{H}$ (1974). Systolic and diastolic blood pressure measurements by transcutaneous Doppler ultrasound in premature infants in critical care nurseries and at closedheart surgery. Anesthesia and Analgesia, 53: 914-918.

7. Steagall HF, Kardon MB \& Kemmerer WT (1968). Indirect measurement of arterial blood pressure by Doppler ultrasonic sphygmomanometry. J oumal of Applied Physiology, 25: 793-798.

8. Colan SD, Fujii THE, Borow KM, MacPherson D \& Sanders S (1983). Noninvasive determination of systolic, diastolic and mean arterial blood pressure in neonates, infants and young children: comparison with central aortic pressure measurements. American J oumal of Cardiology, 52: 867-870.

9. Friesen RH \& Lichtor J L (1981). Indirect measurement of blood pressure in neonates and infants utilizing an automatic noninvasive oscillometric monitor. Anesthesia and Analgesia, 60: 742-745.

10. Reder RF, Dimich I, Cohen ML \& Steinfeld L (1978). Evaluating indirect blood pressure measurement techniques: the comparison of three systems in infants and children. Pediatrics, 62: 326-330.
11. Whyte RK, Elseed AM, Fraser CB, Shineboume EA \& De Swiet M (1975). Assessment of Doppler ultrasound to measure systolic and diastolic blood pressure in infants and young children. Archives of Disease in Childhood, 50: 542-544.

12. Whincup H, Cook DG \& Shaper AG (1989). Measurement of blood pressure in children. British Medical J oumal, 299: 793.

13. Perloff D, Grim C, Flack J, Frohlich ED, Hill $M, M c D o n a l d ~ M \&$ Morgenstern BZ (1993). Human blood pressure determination by sphygmomanometry. Circulation, 88: $2460-2470$.

14. Gustofson TL (1984). True Epistat Manual. 5th edn. Epistat Services, Richardson, TX, USA.

15. De Swiet M, Fayers $P \&$ Shineboume EA (1980). Systolic blood pressure in the population of infants in the first year of life: the Brompton study. Pediatrics, 65 : 1028-1035.

16. Dean AG, Dean J A, Coulombier D, Bredel KA, Smith DC, Burton AH, Dicker RC, Sullivan K, Fagan RF \& Amer TG (1994). Program for Epidemiology on Microcom- 
puters. Centers for Disease Control and Prevention (Epi Info, version 6: the work processing, database and statistics), Atlanta, GA, USA.

17. Diggle J, Liang KY \& Zeger SL (1994). Analysis of Longitudinal Data. Clarendon Press, Oxford, England.

18. Grim CM \& Grim CE (1998). Blood pressure measurement. In: Izzo J r J L \& Black HR (Editors), Hypertension Primer; the Essentials of High Blood Pressure. 2nd edn. American Heart Association, Dallas, TX, USA, 295-298.

19. Task Force on Blood Pressure Control in Children (1987). Report of the Second Task Force on Blood Pressure Control in Children - 1987. Pediatrics, 79: 1-25.

20. Cona CO (1986). Tensión arterial sistolica y diastolica en niños en el primer año de vida. Revista del Hospital de Niños, 28: 156-172.
21. Uhari M (1980). Changes in blood pressure during the first year of life. Acta Paediatrica Scandinavica, 69: 613-617.

22. Kaplan NM (1998). Measurement of blood pressure. In: Kaplan NM (Editor), Clinical Hypertension. 7th edn. Williams \& Wilkins, Baltimore, MD, USA, 19-39.

23. Matsuoka OT, Pinheiro AC, Pascuas DZ \& Leone CR (1996). Evolução dos níveis de pressão arterial sistêmica no período neonatal em recém-nascidos de termo adequados para a idade gestacional. J ornal de Pediatria, 72: 155-158.

24. Nascimento MCVA (1999). Estudo evolutivo do comportamento da pressão arterial, na primeira semana de vida, em recémnascidos a termo, simultaneamente pelos métodos oscilométrico e Doppler. Master's thesis, Universidade Federal de Minas Gerais, Belo Horizonte, MG, Brazil. 25. Lieberman $E$ (1998). Hypertension in childhood and adolescence. In: Kaplan NM (Editor), Clinical Hypertension. 7th edn. Williams \& Wilkins, Baltimore, MD, USA, 407-420.

26. Whincup $\mathrm{H}$, Cook DG, Papacosta $\mathrm{O} \&$ Walker M (1992). Childhood blood pressure, body build, and birth weight: geographical associations with cardiovascular mortality. J ournal of Epidemiology and Community Health, 46: 396-402.

27. Taittonen L, Nuutinen M, Turtinen J \& Uhari M (1996). Prenatal and postnatal factors in predicting later blood pressure among children: Cardiovascular risk in young Finns. Pediatric Research, 40: 627632.

28. O'Sullivan MJ, Keamey J \& Crowley MJ (1996). The influence of some perinatal variables on neonatal blood pressure. Acta Paediatrica, 85: 849-853. 